\title{
Building a Social Media Digital Library: Collection, Management, and Analytics
}

\author{
Hsinchun Chen \\ Director, Artificial Intelligence Lab; \\ McClelland Professor, University of Arizona
}

\begin{abstract}
In this talk I will present the University of Arizona Artificial Intelligence Lab's recent research in Dark Web, Geopolitical Web, and Business Analytics. Based on funding from the NSF and several other US agencies, the AI Lab has developed techniques for collecting, managing and analyzing largescale multilingual and multimedia social media contents of relevance to social, geopolitical, and business applications. Our projects aim to study and understand critical social and business phenomena in the cyber world and real world via a computational, data-centric approach. We aim to collect critical social media content generated by various political and business groups, including web sites, forums, chat rooms, blogs, social networking sites, videos, virtual worlds, etc. A social media digital library and portal system has been developed to manage and access these critical multilingual and multimedia contents. We have also developed advanced multilingual data mining, text mining, and web mining techniques to perform link analysis, content analysis, web metrics (technical sophistication) analysis, sentiment analysis, authorship analysis, and video analysis in our research. Selected case studies in geopolitical domains and business intelligence applications will be discussed.
\end{abstract}

